\title{
Adsorption of Light Alcohols in a High Hydrophobic Metal Azolate Framework
}

\author{
R. M. Madero-Castro, J. M. Vicent-Luna*, and S. Calero*
}

Department of Physical, Chemical, and Natural Systems, Universidad Pablo de Olavide.

Ctra. Utrera km. 1. ES-41013 Seville, Spain. 


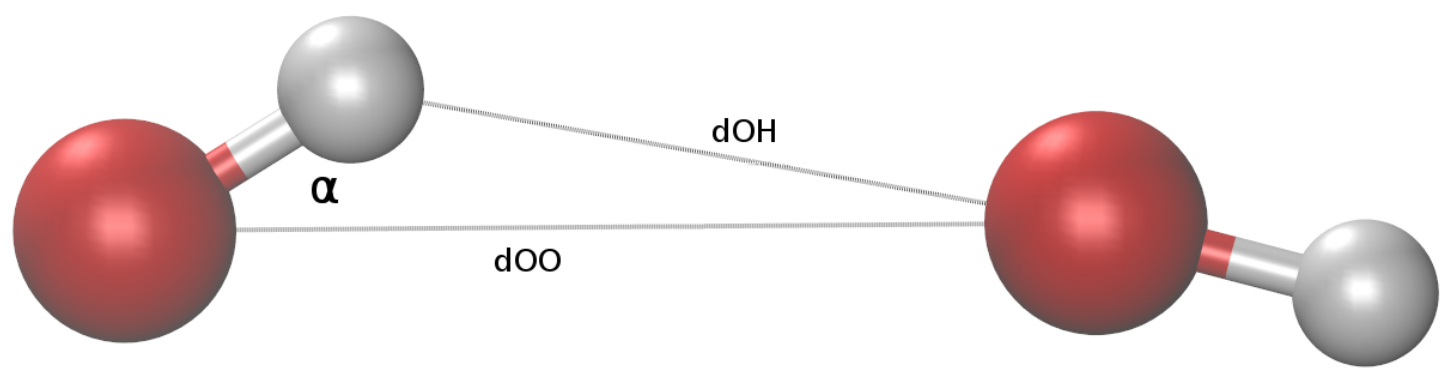

Figure S1. Schematic representation of the HB criterion. Atoms of oxygen are represented by red spheres and hydrogen by white spheres. 

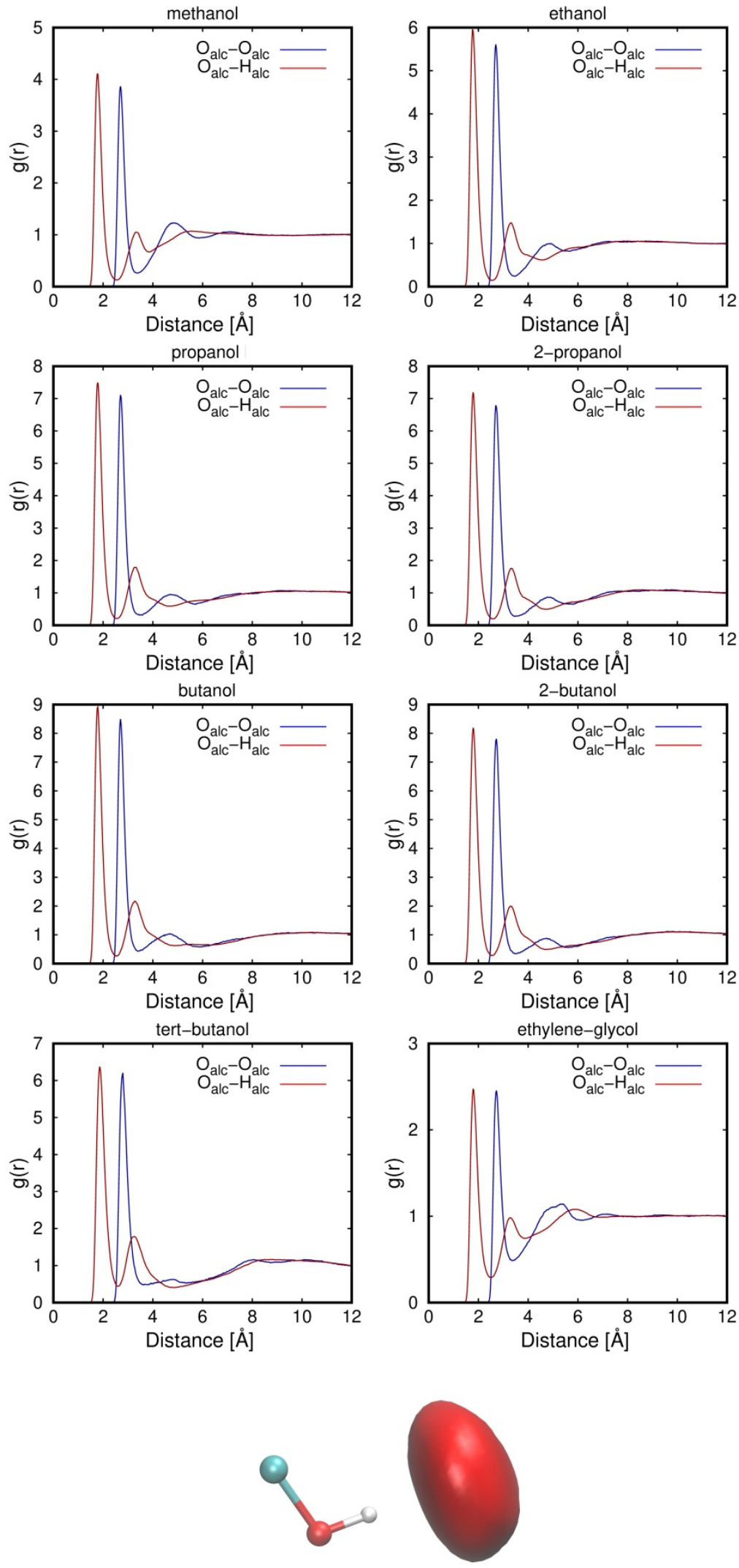

Figure S2. Adsorbate-adsorbate radial distribution functions from MD simulation of each alcohol in the bulk and spatial distribution function of the oxygen atoms than can be connected via hydrogen bonds to a molecule of methanol. 


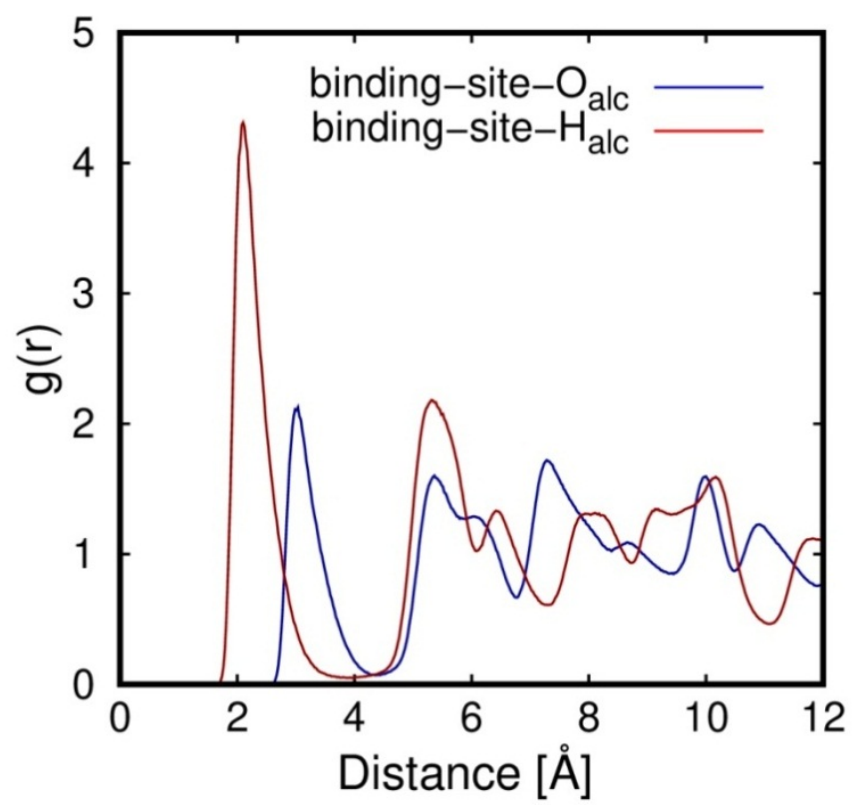

Figure S3. Radial distribution functions of the atoms of oxygen and hydrogen of methanol with the binding-sites of MAF-6.

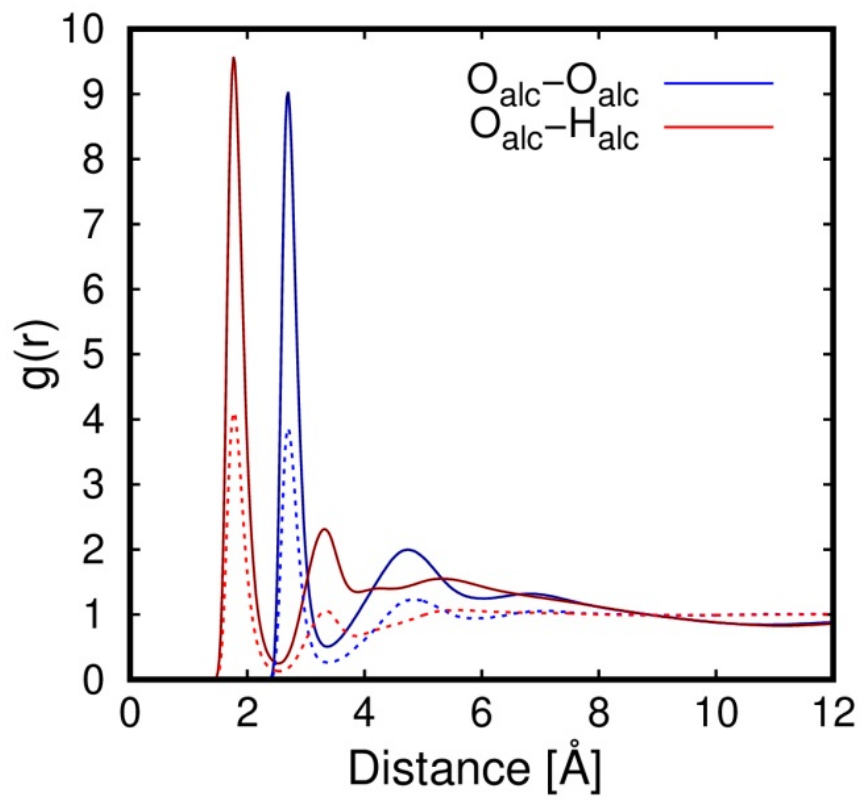

Figure S4. Adsorbate-adsorbate radial distribution functions corresponding to the MD simulation of methanol in the bulk (dashed lines) and confined in the cavities of MAF-6 (solid lines). 
Table S1. Saturation pressure [kPa] of adsorbates calculated using Antoine equation and Peng-Robinson Equation of State.

\begin{tabular}{c|cc|} 
Adsorbate & Antoine Eq. & Peng-Robinson EoS \\
\hline methanol & 16.78 & 15 \\
ethanol & 7.77 & 8 \\
propanol & - & 3 \\
2-propanol & 5.76 & 6 \\
butanol & 0.92 & 1.1 \\
2-butanol & - & 3.2 \\
tert-butanol & 5.51 & 7 \\
ethylene-glycol & - & 0.1
\end{tabular}

Table S2. Average number of hydrogen bonds (nHB) of molecules of alcohol in the bulk.

\begin{tabular}{c|c|} 
Adsorbate & nHB in the bulk \\
\hline methanol & 1.91 \\
ethanol & 1.91 \\
propanol & 1.90 \\
2-propanol & 1.89 \\
butanol & 1.87 \\
2-butanol & 1.83 \\
tert-butanol & 1.68 \\
ethylene-glycol & 3.84
\end{tabular}

